\title{
Implementation of ethics education
}

\author{
Henk ten Have ${ }^{1}$
}

Published online: 26 August 2019

(C) Springer Nature Switzerland AG 2019

In this issue Tom Mishael describes how attempts have been made to introduce ethics teaching in medical schools in India (Mishael 2019). Like in all countries across the world, health care practitioners in India are increasingly confronted with ethical issues in their practices. Over the past decades many efforts have tried to introduce and expand ethics teaching. The need for ethics was stressed by medical associations, councils, and medical colleges. However, ethics teaching programs developed only slowly. As Mishael points out there is now hope for more evolution and implementation of ethics teaching in medical schools. The Medical Council of India (MCI) is now actively promoting a novel teaching program. A significant sign of change is the start of a two-year, full-time Master program in Research Ethics at Yenepoya University in Mangalore. Its Director, Dr. Vina Vaswani, is an active scholar and member of the Editorial Board of this journal.

One basic question is why it is difficult to implement ethics teaching programs. While almost everybody agrees that ethics is important for health care professionals, there are obviously impediments to translate this agreement into educational practices. The MCI has based its proposal on the UNESCO Bioethics Core Curriculum. This is an ethics education program based on the principles of the Universal Declaration of Bioethics and Human Rights. Because there is consensus on the fundamental principles of bioethics in the Declaration, this can be considered as a basis for what should be minimally included in a bioethics course. An Advisory Expert Committee on the Teaching of Ethics, composed of members of the International Bioethics Committee and the World Commission on the Ethics of Scientific Knowledge as well as representatives of the UNESCO Chairs in Bioethics, the Academy of Sciences for the Developing World (TWAS) and the World Medical Association (WMA) developed the proposal with teaching units related to the principles of the Declaration. For each unit, the possible objectives and contents are described, and proposals for teaching materials, resources and assessment methods are provided. The proposal has taken into account the recommendations of a consultation meeting with 30 experts, mainly from developing countries. The proposal has also been tested in a number of universities all over the world (Argentina, Armenia, Belarus, Israel, Japan, Moldova and the Russian

Henk ten Have

tenhaveh@duq.edu

1 Duquesne University Center for Healthcare Ethics, Pittsburgh, PA, USA 
Federation). Based on these experiences, the proposal has been revised and updated. It was translated into Arabic, French, Russian and Spanish. The core bioethics course can assist scholars who want to establish teaching programs in bioethics in various cultures and regions. Also, books with cases from various countries are available to be used in the units of the course.

Nonetheless, an international proposal for bioethics teaching does not automatically leads to its application. More will be needed. Lynette Fernandes and her colleagues from Australia report in this issue how they set up an ethics teaching program for undergraduate students in Arts, Commerce, Design of Science focused on social responsibility in their university (Fernandes et al. 2019). The program was highly appreciated by the students. The key to its success seems to be cooperation and interdisciplinarity. Lecturers were brought together from five disciplines; philosophy, animal biology, anatomy, law, and pharmacology. Such cooperation can overcome one of the impediments to implementation of ethics teaching: the shortage of competent teachers. In order to address this challenge, UNESCO has initiated ethics teacher training courses, to make sure that a new generation of professionals and scientists would be encouraged to teach ethics in a professional manner. Such ethics teacher training has taken place in Romania (2006), Kenya (2007), Slovak Republic (2007), Saudi Arabia (2007), Belarus (2008), Croatia (2010 and 2011), and Serbia (2011). It continues to be organized in Indonesia for example (2019) The focus of the courses is on didactic skills required for ethics teaching rather than on content issues of bioethics. Such training is important but not enough. It is necessary that universities themselves will undertake more efforts to train teachers in ethics. Interdisciplinary cooperation will be a major stimulus to promote programs. This is all the more necessary since research is frequently affected by unethical conduct, as Niraj Shenoy from the USA briefly highlights in this issue (Shenoy 2019).

\section{References}

Fernandes, Lynette B., Nin Kirkham, Anna-Marie Babey, and Dominique Blache. 2019. Implementation of a multi-disciplinary ethics unit. International Journal of Ethics Education 4 (2) in this issue.

Mishael, J.Tom. 2019. Looking back - Looking forward: Ethics finds its place in the medical curriculum in India. International Journal of Ethics Education 4 (2) in this issue.

Shenoy, Niraj. 2019. Professional ethics in academia: Defining the categories of behavior spectrum in matters of unethical conduct. International Journal of Ethics Education 4 (2) in this issue.

Publisher's note Springer Nature remains neutral with regard to jurisdictional claims in published maps and institutional affiliations. 